\title{
Three-body continuum energy correlations in Borromean halo nuclei. III. Short-range external fields
}

\author{
B. V. Danilin, ${ }^{1,}{ }^{*}$ J. S. Vaagen, ${ }^{1}$ T. Rogde,${ }^{1}$ S. N. Ershov,${ }^{1, \dagger}$ I. J. Thompson,,${ }^{2,3}$ and M. V. Zhukov ${ }^{4}$ \\ (RNBT Collaboration) \\ ${ }^{1}$ Department of Physics and Technology, University of Bergen, Bergen, Norway \\ ${ }^{2}$ Physics Department, University of Surrey, Guildford, Surrey GU2 7XH, United Kingdom \\ ${ }^{3}$ Lawrence Livermore National Laboratory, P.O. Box 808, L-414, Livermore, California 94551, USA \\ ${ }^{4}$ Fundamental Physics, Chalmers University of Technology, S-41296 Göteborg, Sweden
}

(Received 5 July 2007; published 28 December 2007)

\begin{abstract}
Energy correlations in transitions from the bound state to the three-body continuum of Borromean halo nuclei are considered. A core $+n+n$ three-body cluster model which reproduces the experimentally known properties of ${ }^{6} \mathrm{He}$ and ${ }^{11} \mathrm{Li}$ has been used to study the low-lying resonances and soft modes. An analysis of the correlated responses in ${ }^{6} \mathrm{He}$ shows that in the case of the narrow three-body $2_{1}^{+}$resonance the transition energy correlations are the same as in the intrinsic correlated structure in $3 \rightarrow 3$ scattering. They differ significantly for wide $2_{2}^{+}, 1_{1}^{+}$resonances, and also for the soft dipole and monopole modes, where, due to the transition operators, the intertwining of the ground state and the three-body continuum plays a significant role.
\end{abstract}

DOI: 10.1103/PhysRevC.76.064612

PACS number(s): 21.45.+v, 24.10.-i, 24.30.Gd, 27.20.+n

\section{INTRODUCTION}

This article continues a series of papers which investigates the three-body continuum and transition properties of Borromean halo nuclei, with particular reference to ${ }^{6} \mathrm{He}$ as a case study. The earliest articles [1] examined simple transition responses, summed over all final states. More recently we have studied general features of the spatial two-dimensional densities [2] and intrinsic energy correlations in $3 \rightarrow 3$ scattering [3], again exemplified by the continuum of ${ }^{6} \mathrm{He}$. Three-body core $+n+n$ dynamics and the Schrödinger threebody equation, solved within the method of hyperspherical harmonics (HH) (see Refs. [1-5]), were used consistently for both bound and continuum states. For ${ }^{6} \mathrm{He}$ a 'realistic' $\alpha n$ interaction $[1,4]$ with purely repulsive $s$-wave component and the GPT $n n$ interaction [6] were used.

The three-body model has been successfully tested in calculations of bound and lowest excited states of the $A=6$ nuclei $[1,4]$, for the calculation of inelastic ${ }^{6} \mathrm{He}\left(n, n^{\prime}\right)$ and charge-exchange ${ }^{6} \mathrm{Li}(n, p)$ reactions to the ${ }^{6} \mathrm{He}$ continuum [7,8], and for the inelastic ${ }^{11} \operatorname{Li}\left(p, p^{\prime}\right)$ reaction [9]. The continuum and ground state (g.s.) wave functions were more recently also used for studying elastic and inelastic breakup of ${ }^{6} \mathrm{He}$ on ${ }^{12} \mathrm{C}$ and $\mathrm{Pb}$ targets [10] under the kinematically complete conditions of GSI experiments [11]. Very recently [12], angular and energy correlations for ${ }^{6} \mathrm{He}$ have been measured and compared with the predictions of our model.

With one-step break-up reaction theories such as DWBA, applicable in the high energy regime, or the semiclassical

\footnotetext{
*Permanent address: Russian Research Center "The Kurchatov Institute," RU-123182 Moscow, Russia.

†Permanent address: Joint Institute for Nuclear Research, RU141980 Dubna, Russia.
}

method for electromagnetic dissociation, involving transitions from the g.s. to the three-body continuum, we have found the transition densities to be the decisive ingredient for the energy dependence of the break-up cross section. The particular reaction mechanism scales the transition amplitudes and slightly distorts the shape of the cross section. The transition densities are also influenced by intertwined correlations where both the g.s. and the three-body continuum contribute.

For three-body nuclear Borromean systems we can never directly observe the intrinsic structure of the continuum since the $3 \rightarrow 3$ nuclear scattering seems impossible to perform experimentally. In the molecular case this was, however, recently performed for caesium atoms in a trap [13]. Thus we have to deal with the responses (in the simplest case an integrated and squared transition density), generated by transition operators from the g.s., in which case the g.s. acts as a filter. Also, in contrast to transitions from compact bound states, the range of impact parameters acceptable in $3 \rightarrow 3$ scattering is limited by a maximal hypermoment $K<\sqrt{2 m E / \hbar^{2}}\langle\rho\rangle$ where $E$ is the continuum energy, $m$ the nucleon mass and $\left\langle\rho^{2}\right\rangle=\left(A_{c}+2\right) R_{A_{c}+2}^{2}-A_{c} R_{A_{c}}^{2}$. The $\left\langle\rho^{2}\right\rangle^{1 / 2}$ is the rms hyperradius of the bound state expressed via rms radii of the core $\left(R_{A_{c}}\right)$ and of the halo nucleus $\left(R_{A_{c}+2}\right)$ [14], and is also the three-body impact parameter in the $3 \rightarrow 3$ scattering case [3].

The difference between the intrinsic structure of the continuum and responses is most dramatically seen if the binary interactions are set to zero for the continuum wave functions: this is the no-final-state-interaction (NoFSI) case often used for estimates. The continuum structure would then consist of plane waves antisymmetrized between the halo nucleons, with zero phase shifts, and there would never be any identifiable discrete continuum 'states' apart from the nonresonant and no-interacting continuum. Even in this case, there might be relatively narrow peaks in the continuum response from the g.s., especially if long-range transition operators such as $r$ or 
$r^{2}$ are used. But it is clear that although such peaks reflect a time delay of excitation processes, they should never be identified as intrinsic continuum 'states' with widths and lifetimes. The correlations (or momentum distributions) in the g.s. can also resemble the narrow momentum distributions of fragments measured in break-up reactions (and they can even be peaked at low excitation energies), which in reality can be caused by low-lying resonances in the three-body continuum $[8,15]$.

In the current article, we analyze the transition energy correlations for the lowest $J^{\pi}$ in the Borromean continuum when written as functions of the energies in the different sets of Jacobi coordinates. Only nuclear type transitions are included in this article. We give an analysis of the one- and twodimensional transition energy correlations in the three-body problem, pointing out the simplest analytical properties, which can be revealed in kinematically complete experiments. This gives possibilities for discriminating between experimental and/or theoretical ambiguities. In future work we will also discuss the angular/energy correlations in the ${ }^{6} \mathrm{He}$ continuum. In all cases we use realistic potentials for the ${ }^{6} \mathrm{He}$ case, and the Feshbach method [16] for reducing a basis from up to hyperangular momentum $K \leqslant 40(41)$ for three interacting bodies, to an active space of $K \leqslant 10(11)$ for even and odd parities respectively. The $0^{+}$state in ${ }^{11} \mathrm{Li}$ within the spinless core $+n+n$ approach, and the dipole nuclear response have been obtained in our P2 model $[9,17]$.

\section{ENERGY CORRELATIONS IN TRANSITIONS TO THREE-BODY CONTINUUM}

The three-body problem contains two extreme spatial scales for the three interacting bodies. The first (a) is a short-range scale, with size about the sum of the radii of the binary interactions, and which produces a compact spatial structure such as a true three-body resonance. The most remarkable feature of a 'true' three-body resonance is that it exists in the configurations with the lowest hyperangular momenta, which corresponds to the three particles interacting while close to each other.

A second spatial scale (b) arises from the phenomenon of a long-range effective three-body interaction with a range about the sum of the scattering lengths in the binary subsystems. This is responsible for the Efimov effect [18] and for a spectral compression near the three-body threshold. In general (b) reflects the possibility of some two interacting particles to 'feel' the presence of the third one at a distance up to the scattering length. When we deal with a Borromean halo, the neutron-neutron interaction with scattering length $\sim 16 \mathrm{fm}$ is decisive for this effect ('continuum pairing'). Additionally, in the ${ }^{11} \mathrm{Li}$ case [19] the presence of an intruder virtual $s$-state in ${ }^{10} \mathrm{Li}$ gives a large (but still not experimentally fully determined) scattering length, which contributes essentially to its unusually large matter radius.

Another possibility is that a large correlation distance could arise from a long-living binary resonance propagating to a large distance. In this case there is no concentration of wave function inside the region of interaction of all particles, but a long range spreading of correlations.
One can expect four main physical sources for amplification of transitions, partly in correspondence with the possible amplification of cross sections for $3 \rightarrow 3$ scattering [3]:

(i) True three-body resonances, which are due to interaction of all three particles in the interior domain;

(ii) A long lived binary resonance in one of the constituent pairs;

(iii) The response of an extended system to long-range transition operators used to excite the continuum;

(iv) Resonances due to strong coupling between channels (CC resonance in a few channels) or a parametric resonance in quantum diffusion with complex coefficients [20,21].

Resonance criteria are as in the two-body case: (A) a concentration of the wave function in the interior region (except for barrier top and virtual-state cases), and that (B) the existence and properties of any intrinsic resonant state should not depend on the excitation mechanism (electromagnetic, strong or weak interaction, etc.) that produces it.

To study the three-body problem, translationally-invariant coordinates are used. After separation of the center of mass motion, the intrinsic excitation properties can be displayed in two 'binary' Jacobi subsystems (any pair of particles and the relative motion of this pair relative to the third constituent). A way to gain an insight into these degrees of freedom is to analyze their correlation properties.

In the Appendices of Refs. [2] and [3] we summarized the Jacobi spatial $\mathbf{x}, \mathbf{y}$ and relative momentum $\mathbf{k}_{x}, \mathbf{k}_{y}$ coordinates necessary to describe the bound and scattering states of three-body systems consisting of a spinless core and two halo nucleons. We henceforth use the same notations for our three-body wave functions and all variables.

\section{A. Defining energy correlations}

We start from the simplest (but very important) characteristics such the angular-averaged energy correlations. From the theoretical point of view, it is convenient to use Jacobi coordinates to describe internal excitations of the system, i.e., a coordinate for the relative motion between two particles (distance $\mathbf{x}_{i}$, conjugate momentum $\mathbf{k}_{x i}$, energy $\epsilon_{x_{i}}=\hbar^{2} k_{x i}^{2} / 2 m$ ) and another between the c.m. of these particles and the third one $\left(\mathbf{y}_{i}, \mathbf{k}_{y i}, \epsilon_{y_{i}}\right)$. In both cases the Jacobi coordinates are related to the physical distances by an appropriate mass scaling. Of the three Jacobian systems $\{i\}$ we will only use two because of the identity of the two halo neutrons. In one Jacobi system (T) we use the neutron-neutron relative motion energy $E_{n n}=\epsilon_{x_{i}}$ and the energy between the core (C) and the c.m. of the halo neutrons $E_{(n n)-C}=\epsilon_{y_{i}}$. In the other Jacobi system (Y) we examine the neutron-core relative motion energy $E_{C n}=\epsilon_{x_{j}}$ and the energy between the c.m. of the core-neutron and the second neutron $E_{(C n)-n}=\epsilon_{y_{j}}$. The total continuum energy $E_{\kappa}$ and the three-body phase space $\sqrt{\epsilon_{x} \epsilon_{y}} d \epsilon_{x} d \epsilon_{y}$ are invariant with respect to the choice of a binary partition $\left(E=\epsilon_{x}+\epsilon_{y}=E_{n n}+E_{(n n)-C}=E_{C n}+\right.$ $E_{(C n)-n}$ and $\left.k_{x}^{2} d k_{x} k_{y}^{2} d k_{y}=2\left(m / \hbar^{2}\right)^{3} \sqrt{\epsilon_{x} \epsilon_{y}} d \epsilon_{x} d \epsilon_{y}\right)$. 
In practice all possible information about the three-body continuum structure is extracted from nuclear reactions, where however the interplay of reaction mechanism and the structure of initial and final states makes this task difficult. Only for situations at sufficiently high energy, where we can assume one-step nuclear reactions, or reactions with well-defined mechanisms (e.g., electromagnetic), can we give reasonably simple connections between the nuclear structure and reaction observables. Thus the expression for the differential cross section (Eq. (2.1) from [7]) for elastic breakup, contains the transition matrix element

$$
T_{f i}=\left\langle\chi_{0}^{(-)}\left(\mathbf{k}_{f}\right) \Phi_{0} \Psi^{(-)}\left(\mathbf{k}_{x}, \mathbf{k}_{y}\right)\left|\sum_{p, t} V_{p t}\right| \Psi_{0} \Phi_{0} \chi_{0}^{(+)}\left(\mathbf{k}_{i}\right)\right\rangle .
$$

In this matrix element (brackets \langle\rangle means integration over all spatial coordinates) the product of the internal target wave function $\Phi_{0}$ with the distorted waves $\chi_{0}^{+}$and $\chi_{0}^{-}$corresponding to relative motion in the initial and final channels is folded with the transition matrix element between g.s. and the threebody continuum of the Borromean projectile $\left\langle\Psi^{(-)}\left(\mathbf{k}_{x}, \mathbf{k}_{y}\right)\right.$ | $\sum_{p, t} V_{p t}\left|\Psi_{0}\right\rangle$. The $V_{p t}$, the interaction between projectile and target nucleons, is thus integrated over the distorted waves and the internal target wave function $\Phi_{0}$. After integration over the scattering angle of the full c.m. motion, we obtain an approximately factorized expression for the differential cross section for fragmentation in the center of mass of the projectile:

$$
d^{6} \sigma \sim \sum_{J_{f}, j, l, s} F\left(E_{i}, E_{f}\right)_{J_{f}} \cdot d B^{J_{f}}(j l s) d^{3} k_{x} d^{3} k_{y} .
$$

This factorization follows because $\sum V_{p t}$ is composed of effective nucleon-nucleon short-range nuclear interactions $V_{p t}$ between projectile $(p)$ and target $(t)$ nucleons, which for small excitation energies can be treated as being approximately of zero range. The Coulomb long range pair interaction is however also contained. The reaction dynamics is mainly determined by the transferred total angular momentum $j$, the orbital angular momentum $l$, the spin $s$ and the transferred (linear) momentum. The functions $F\left(E_{i}, E_{f}\right)$ in the above equation are smooth functions of the excitation energy $E$ in the limit of $E_{i}, E_{f} \gg E$, when a one-step process dominates.

The double-differential correlated response, after integrating over the four-dimensional angular parts of the momenta of the scattered particles, and averaging over the initial $M$-states and summing over final states (we omit spin variables for simplicity), becomes

$$
\frac{d^{2} B^{J_{f}}(j l s)}{d \epsilon_{x} d \epsilon_{y}} \propto \sqrt{\epsilon_{x} \epsilon_{y}} \int\left|\left\langle J_{f} \pi_{f}\left\|\hat{T}_{l s j \tau}(r)\right\| J_{i} \pi_{i}\right\rangle\right|^{2} d^{3} r d \hat{\mathbf{k}}_{\mathbf{x}} d \hat{\mathbf{k}}_{\mathbf{y}} .
$$

The bracket \langle\rangle designates integration over internal coordinates and summation over spins, and $r$ corresponds to the external field (see below) exciting the projectile. This expression includes the cases of long-range electromagnetic and shortrange nuclear correlated responses. The zero-range nuclear transition operators $T_{l s j m_{j} \tau}^{\mathcal{T}}$ and Coulomb multipole operators $T_{l m}^{\mathcal{E}}$ for point-like particles, referred to the projectile center of mass, are given by

$$
\hat{T}_{l m}^{\mathcal{E}}(r)=\sum_{i=1}^{3} z_{i} \frac{\delta\left(r-r_{i}\right)}{r_{i}^{2}} r_{i}^{l} Y_{l m}\left(\hat{r}_{i}\right)(l>0)
$$

and

$$
\hat{T}_{l s j m_{j} \tau}^{\mathcal{T}}(r)=v_{0} \sum_{i=1}^{3} \frac{\delta\left(r-r_{i}\right)}{r_{i}{ }^{2}}\left[Y_{l}\left(\hat{r}_{i}\right) \otimes \sigma_{s}(i)\right]_{j m_{j}} t_{\tau}(i) .
$$

Here $z_{i}=e Z_{i}$ are the electric charges and $v_{0}$ is the strength of the external field. Inelastic and charge-exchange reactions correspond to isospin transitions $\mathcal{T}=0$ and 1 , respectively.

In the generic one-step transition amplitude (using the transition density for the nuclear operator with $v_{0}=1$ )

$$
T_{f i}(r)=\sum_{J_{f}, j, l, s}\left\langle J_{f} \pi_{f}\left|\hat{T}_{l s j \tau}^{\mathcal{T}}\right| J_{i} \pi_{i}\right\rangle,
$$

we really should use a full final state wave function, and not the asymptotic form as in [3], as this wave function includes the intrinsic properties of the continuum. One alternative to the solution of differential equations is the more transparent solution of a Lippmann-Schwinger integral equation where the sum of a plane wave and of the scattered (hyper)spherical wave is a solution in the whole space. Therefore, in breakup type reactions the correlated response of Eq. (3) should contain three terms: the "no final state interaction (NoFSI)" term, the FSI term and an interference term. To extract conclusions about three-body properties, in particular about binary subsystems, we should inspect both the 'NoFSI' (as these often resemble g.s. energy/momentum correlations) as well as the FSI transitions, where the intrinsic properties of continuum are intertwined with the g.s. correlations and where some of the observables are influenced by the range of the transition operator.

It should be noted here that it is enough to calculate the transition amplitude in one Jacobi system, the most suitable for antisymmetrization. Amplitudes in other systems can then be obtained by kinematic rotations [22].

Generally, for a 'true' three-body resonance with given $J^{\pi}$, the $3 \rightarrow 3$ scattering amplitude has the analytic property [23]

$$
A^{J}\left(E, \Omega_{5}^{\rho}, \Omega_{5}^{\kappa}\right) \propto \frac{A^{J}\left(\Omega_{5}^{\rho}, \Omega_{5}^{\kappa}\right)}{E-\left(E_{0}-i \Gamma_{\mathrm{T}} / 2\right)},
$$

where $E$ is the total energy calculated from the three-body threshold, and $E_{0}$ and $\Gamma_{\mathrm{T}}$ are the position and total width of the resonance. This agrees with the resonant factorization of the wave functions in the interior region, pointed out in our previous article [2], and therefore the transition amplitude will have the same resonant property.

In the $i$ th Jacobi system and for small $\left(\epsilon_{x i}, \epsilon_{y i}\right)$ values, the behavior of the correlation function is defined by the phase volume $\sim \sqrt{\epsilon_{x i} \epsilon_{y i}}$ and the lowest partial angular momenta $l_{x}, l_{y}$ of the state $J^{\pi}$ via kinematical multipliers $\epsilon_{x i}^{l_{x}} \epsilon_{y i}^{l y}$ in the product of the hyperangular parts of the hyperspherical functions, $\psi_{K}^{l_{x} l_{y}}\left(\alpha_{i}\right) \psi_{K^{\prime}}^{l_{x} l_{y}}\left(\alpha_{i}\right),\left(\sin ^{2} \alpha_{i}=\epsilon_{x i} / E\right)$. The HH has the structure $\psi_{K}^{l_{x} l_{y}}\left(\alpha_{i}\right) \simeq(\sin \alpha)^{l_{x}}(\cos \alpha)^{l_{y}} P_{\left(K-l_{x}-l_{y}\right) / 2}^{l_{x}+1 / 2, l_{y}+1 / 2}(\cos 2 \alpha)$, where 
$P_{n}^{a, b}$ are Jacobi polynomials (similar to Legendre polynomials $P_{n}^{a}$ in the spherical harmonics case).

For Borromean nuclei represented as core $+n+n$, the correlation function in the $\mathbf{Y}$ coordinate system, with $\epsilon_{x i}=$ $E_{C n}$ and $\epsilon_{y i}=E_{(C n)-n}$, should be almost symmetrical about the hyperangle $\alpha_{Y} \sim 45^{\circ}$ because of the antisymmetrization between the halo nucleons, but with a deviation that depends on the ratio of the reduced masses for $x$ and $y$ motion. The deviation from this symmetry is a recoil effect, which disappears in the limit of an infinitely heavy core.

Integrating over $\epsilon_{x i}$ or $\epsilon_{y i}$, we obtain the binary energy correlations, which are subject for various conclusions in discussions of experimental data. In Sec. III we discuss two kinds-NoFSI and FSI — of nuclear type energy correlations in ${ }^{6} \mathrm{He}$. First we outline some general features.

\section{B. Energy correlations for NoFSI}

If we use a (halo neutron) antisymmetrized three-body plane wave [1] as the final state, the expression (3) for the correlated response gives the g.s. energy (momentum) correlations for the $T_{000}$ transition operator, a Fourier-like transform, and this is often used for Serber-type reaction mechanisms. Generally this reflects the uncertainty principle and might reveal the spatially correlated structure of the g.s. (or g.s. momentum correlations).

Higher transition multipoles also serve for calculating 'NoFSI' nuclear break-up reaction cross sections with a zero-range nuclear perturbation (5). They reflect only the g.s. peripheral correlations and are treated as a reference for the case when the FSI is switched on.

\section{FSI energy correlations}

We will focus on the low excitation energy breakup of halo nuclei, which only involves a moderately large number of final partial waves. An expression which can be derived from general considerations [7], and which exhibits the main features of the correlation function (3) with definite $J_{f}$, is

$$
\begin{aligned}
\frac{d^{2} \sigma(3)}{d \epsilon_{x i} d \epsilon_{y i}} \propto & \sum_{l_{x}, l_{y}}\left(\epsilon_{x i} / E\right)^{l_{x}}\left(\epsilon_{y i} / E\right)^{l_{y}} \sqrt{\epsilon_{x i} \epsilon_{y i}} \\
& \times\left|\sum_{K, K^{\prime}} R_{K l_{x} l_{y}} R_{K^{\prime} l_{x} l_{y}} C_{K, K^{\prime}, l_{x}, l_{y}}^{J_{f}}\left(\epsilon_{x i} / E, \epsilon_{y i} / E\right)\right|^{2} .
\end{aligned}
$$

This expression involves an incoherent summation over $l_{x}$ and $l_{y}$ after integration over the angles of the $\mathbf{k}_{x}, \mathbf{k}_{y}$ momenta. We have to underline the behavior of the cross section at small $\epsilon_{x i} / E, \epsilon_{y i} / E$, and have especially extracted the dependence on $\left(\epsilon_{x i} / E\right)^{l_{x}}\left(\epsilon_{y i} / E\right)^{l y}$ to a polynomial factor $C_{K, K^{\prime}, l_{x}, l y}^{J}\left(\epsilon_{x i} / E, \epsilon_{y i} / E\right)$ which is nearly a constant. The factors $R_{K l_{x} l_{y}} R_{K^{\prime} l_{x} l_{y}}$ contain the spin-angular parts of the reduced matrix element and the hyperradial integrals. Such factorization follows from the multiplicative structure of the hyperangular part

$$
\psi_{K}^{l_{x} l_{y}}\left(\alpha_{\kappa}\right)=N_{K}^{l_{x} l_{y}}\left(\sin \alpha_{\kappa}\right)^{l_{x}}\left(\cos \alpha_{\kappa}\right)^{l_{y}} P_{\left(K-l_{x}-l_{y}\right) / 2}^{l_{x}+1 / 2, l_{y}+1 / 2}\left(\cos 2 \alpha_{\kappa}\right)
$$

of the hyperspherical harmonic $Y_{K \gamma}\left(\Omega_{5}^{\kappa}\right)$, where $\gamma=$ $\left(l_{x}, l_{y}, L\right)$ denotes the Jacobi orbital and total orbital angular momenta, and which depends on the momentum angles $\Omega_{5}^{\kappa}$ in hyperspherical coordinates [2,3]. The hyperangle $\alpha_{\kappa}$ is defined by the relations $\epsilon_{x}=E \sin ^{2} \alpha_{\kappa}$ and $\epsilon_{y}=E \cos ^{2} \alpha_{\kappa}$

The hyperradial matrix elements $R_{K l_{x} l_{y}}(E)$, which in the case of $3 \rightarrow 3$ scattering are regular at $E \rightarrow 0$, diminish in low inverse powers of $E$ at high energy. The kinematic singularity $\kappa^{-5 / 2}$ in the scattering wave function [3], where $\kappa \propto \sqrt{E}$, is compensated by the $\kappa^{K+5 / 2}$ dependence of its regular part.

Energy correlations for a narrow three-body resonance. The similarity of the energy dependence in the analytical structure of the amplitude of a three-body resonance wave function in the internal region [2] and the scattering amplitude (7), which characterizes the asymptotic behavior, implies that expressions for the correlation functions will be similar to the intrinsic correlations [3] in the vicinity of the resonance ridge $E_{0}=\epsilon_{x i}+\epsilon_{y i}$. The profile of the ridge could however vary because, in the transition matrix element, the g.s. plays the role of an angular-spin filter for different partial components.

For qualitative and semiquantitative understanding we can apply the analytic properties of the transition amplitudes in the region of a true three-body resonance $\left(E_{0}, \Gamma_{\mathrm{T}}\right)$. In order to have a reference case where analytical estimates are possible, the following approximation is useful. Inspired by the close analogy between partial wave expansions in the two-body and three-body problems, we consider, in first approximation, only the diagonal parts of the continuum wave function. In the interior region, (for given $J^{\pi}$ ) the resonant three-body wave function takes the form given in [2] (neutron spins are not indicated explicitly and the kinematic singularity $1 / \kappa^{5 / 2}$ is included):

$$
\begin{aligned}
& \psi\left(\rho, \Omega_{5}^{\rho} ; E, \Omega_{5}^{\kappa}\right) \\
& \propto \frac{1}{(\kappa \rho)^{5 / 2}} \sum_{K, \gamma} G_{K \gamma}(E) \psi_{K \gamma}^{R}(\rho) Y_{K \gamma}\left(\Omega_{5}^{\rho}\right) Y_{K \gamma}\left(\Omega_{5}^{\kappa}\right),
\end{aligned}
$$

with

$$
\left|G_{K \gamma}(E)\right|^{2}=\frac{\Gamma_{K \gamma}}{\left(E-E_{0}\right)^{2}+\Gamma_{\mathrm{T}}^{2} / 4} .
$$

The energy $E_{0}$ is the position of the resonance, $\Gamma_{K \gamma}$ its partial width, and $\Gamma_{\mathrm{T}}$ the total width while $\psi_{K \gamma}^{R}(\rho)$ is the energyindependent internal part of the resonant wave function. Eq. (11) shows that the dependence of a three-body resonance on $E$ is of pole character typical for an ordinary Breit-Wigner resonance.

In a simplest approximation, both the three-body 'partial width' and the total width are defined with rather high accuracy [1] by

$$
\Gamma_{\mathrm{T}}=\int\left|\left\langle\Psi_{c}|V| \Psi_{R}\right\rangle\right|^{2} \delta\left(E_{R}-E\right) d \rho_{f}=\sum_{K \gamma} \Gamma_{K \gamma},
$$


where

$$
\begin{aligned}
\Gamma_{K \gamma}= & \frac{m}{\hbar^{2} \kappa} \mid i^{K} \sqrt{\kappa} \sum_{K^{\prime} \gamma^{\prime}} \int J_{K+2}(\kappa \rho) V_{K \gamma, K^{\prime} \gamma^{\prime}} \\
& \times\left.(\rho) \chi_{K^{\prime} \gamma^{\prime}}^{R}(\rho) \sqrt{\rho} d \rho\right|^{2},
\end{aligned}
$$

where $d \rho_{f}$ is the three-body phase volume, $\Psi_{R}$ in the general formula is replaced by the unit-normalized internal part $(\rho \leqslant R)$ of the resonance hyperradial wave function $\chi_{K^{\prime} \gamma^{\prime}}^{R}(\rho)$, and where $\Psi_{c}$ is a Bessel function from the three-body plane wave.

In the case of a resonance near threshold, the component with the minimal value $K_{0}$ of the hypermoment dominates in the scattering wave function (10). Using this one-component $\left(K_{0}\right)$ expression for the continuum wave function in the transition matrix (1) leads to a factorized expression for the inelastic break-up cross section. The double differential cross section (3) takes the form

$$
\begin{aligned}
\frac{d^{2} \sigma}{d \epsilon_{x} d \epsilon_{y}} & \propto E^{-5 / 2} \sqrt{\epsilon_{x} \epsilon_{y}} \sum_{l_{x} l_{y}}\left|G_{K_{0} \gamma}(E)\right|^{2}\left|\psi_{K_{0}}^{l_{x} l_{y}}\left(\alpha_{\kappa}\right)\right|^{2} \\
& =\frac{\sqrt{\epsilon_{x} \epsilon_{y}} \sum_{l_{x} l_{y}} \Gamma_{K_{0} l_{x} l_{y}}\left|\psi_{K_{0}}^{l_{x} l_{y}}\left(\alpha_{\kappa}\right)\right|^{2}}{E^{5 / 2}\left[\left(\epsilon_{x}+\epsilon_{y}-E_{0}\right)^{2}+\Gamma_{\mathrm{T}}^{2} / 4\right]} .
\end{aligned}
$$

Energy correlations for a three-body resonance near the three-body threshold. If the resonance is not so narrow, i.e., $\Gamma_{\mathrm{T}} \sim E_{0}$, the energy dependences of the 'partial widths' $\Gamma_{K_{0}} l_{x} l_{y}$ and total width $\Gamma_{\mathrm{T}}$ have to be taken into account. At low energy the width, as a function of energy, mainly depends on the three-body phase volume $\sim E^{2}$ and on the hypermoment $K_{0}$ as $\Gamma_{K_{0} l_{x} l_{y}} \propto \Gamma_{0} E^{2+K_{0}}$ for some constant $\Gamma_{0}$. For the state $J^{\pi}$ with lowest $K_{0}$ we obtain the form

$$
\begin{aligned}
\frac{d^{2} \sigma}{d \epsilon_{x} d \epsilon_{y}} \propto & \frac{\sqrt{\epsilon_{x} \epsilon_{y}}\left(\epsilon_{x}+\epsilon_{y}\right)^{-1 / 2+K_{0}}}{\left(\epsilon_{x}+\epsilon_{y}-E_{0}\right)^{2}+\Gamma_{0}^{2}\left(E / E_{0}\right)^{4} / 4} \\
& \times \sum_{l_{x}, l_{y}}\left(\epsilon_{x} / E\right)^{l_{x}}\left(\epsilon_{y} / E\right)^{l_{y}},
\end{aligned}
$$

where a simple parametrization of the energy dependence is used for the total width: $\Gamma_{\mathrm{T}}=\Gamma_{0}\left(E / E_{0}\right)^{2}$. Integration of Eq. (15) over hyperangle $\alpha_{\kappa}$ using $\epsilon_{x}=E \sin ^{2} \alpha_{\kappa} ; \epsilon_{y}=$ $E \cos ^{2} \alpha_{\kappa}$ and $d \epsilon_{x} d \epsilon_{y}=2 E d E \sin \alpha_{\kappa} \cos \alpha_{\kappa} d \alpha_{\kappa}$ gives an asymmetric resonance shape in the excitation function for the three-body decaying state:

$$
\frac{d \sigma}{E d E} \sim \frac{E^{1 / 2+K_{0}}}{\left(E-E_{0}\right)^{2}+\frac{1}{4} \Gamma_{0}^{2}\left(E / E_{0}\right)^{4}} .
$$

Note that for shape (16), because of the proximity to the energy threshold, neither the position $E_{m}$ of the maximum or its width $\Gamma^{\prime}$ correspond to the pure Breit-Wigner conditions of $E_{m}=E_{0}$ and $\Gamma^{\prime}=\Gamma_{0}$. It is possible to find $E_{m}$ in Eq. (16) by setting the derivative of the right hand side to zero, but it is simpler to find the minimum of the denominator in Eq. (16) , which gives almost the same result. Writing the denominator $f(E)$ in a Taylor series around the minimum, $f(E)=$ $f\left(E_{m}\right)+f^{\prime \prime}\left(E_{m}\right)\left(E-E_{m}\right)^{2} / 2$, we find the relations $E_{m} \leqslant E_{0}$ and $\Gamma^{\prime}=\sqrt{8 f\left(E_{m}\right) / f^{\prime \prime}\left(E_{m}\right)}<E_{0} / 2$ for any values of the parameters $E_{0}$ and $\Gamma_{0}$.
As an example, we consider hypothetical monopole and dipole resonances. For a $0^{+}$state, $K_{0}=l_{x}=l_{y}=0$ in both $\mathbf{T}$ and $\mathbf{Y}$ systems, and the cross sections (15) are symmetric with respect to $\left(\epsilon_{x}, \epsilon_{y}\right)$ permutations and are the same for both Jacobi systems. For $1^{-}$the hypermoment $K_{0}=1$, and the angular momenta $\left(l_{x}, l_{y}\right)$ take values $(0,1)$ and $(1,0)$. Since the two halo neutrons on average attract (repulse) each other for $s$-wave ( $p$-wave) motion, the component with $\left(l_{x}, l_{y}\right)=(0,1)$ is expected to dominate for dipole excitation in $\mathbf{T}$ and gives a cross section asymmetric in $\left(\epsilon_{x}, \epsilon_{y}\right)$. In $\mathbf{Y}$ this cross section corresponds to motion with angular momenta $(0,1)$ and $(1,0)$ with approximately equal weights, and the shape of cross section (15) is close to symmetric.

From Eq. (15) it follows that the contour plot of the cross section $\Phi\left(\epsilon_{x}, \epsilon_{y}\right)=d^{2} \sigma / d \epsilon_{x} d \epsilon_{y}$ has special patterns in the $\left(\epsilon_{x}, \epsilon_{y}\right)$-plane for $\mathbf{T}$ and $\mathbf{Y}$ in the presence of a three-body resonance. A quantitative tool, which can be useful for comparisons with the real cross sections, is the trajectory of the cross section ridge. This trajectory follows the curve $\epsilon_{y}=\epsilon_{y}\left(\epsilon_{x}\right)$ which is defined by an extremum of the directional derivative of the function $\Phi\left(\epsilon_{x}, \epsilon_{y}\right)$ in the $\left(\epsilon_{x}, \epsilon_{y}\right)$-plane. In the region where the cross section is not small, the trajectory can be well approximated by a solution of the simpler equation $\left.\left(d \Phi / d \epsilon_{x}^{\prime}\right)\right|_{\epsilon_{y}^{\prime}=\text { const }}=0$, which can be obtained by a rotation of $\pi / 4$ of the initial coordinate system: $\epsilon_{x}^{\prime}=\left(\epsilon_{x}+\epsilon_{y}\right) / \sqrt{2}, \epsilon_{y}^{\prime}=$ $\left(-\epsilon_{x}+\epsilon_{y}\right) / \sqrt{2}$.

Figure 1(b) shows the contour plot, obtained from Eq. (15) with parameters $E_{0}=0.25 \mathrm{MeV}$ and $\Gamma_{0}=0.3 \mathrm{MeV}$
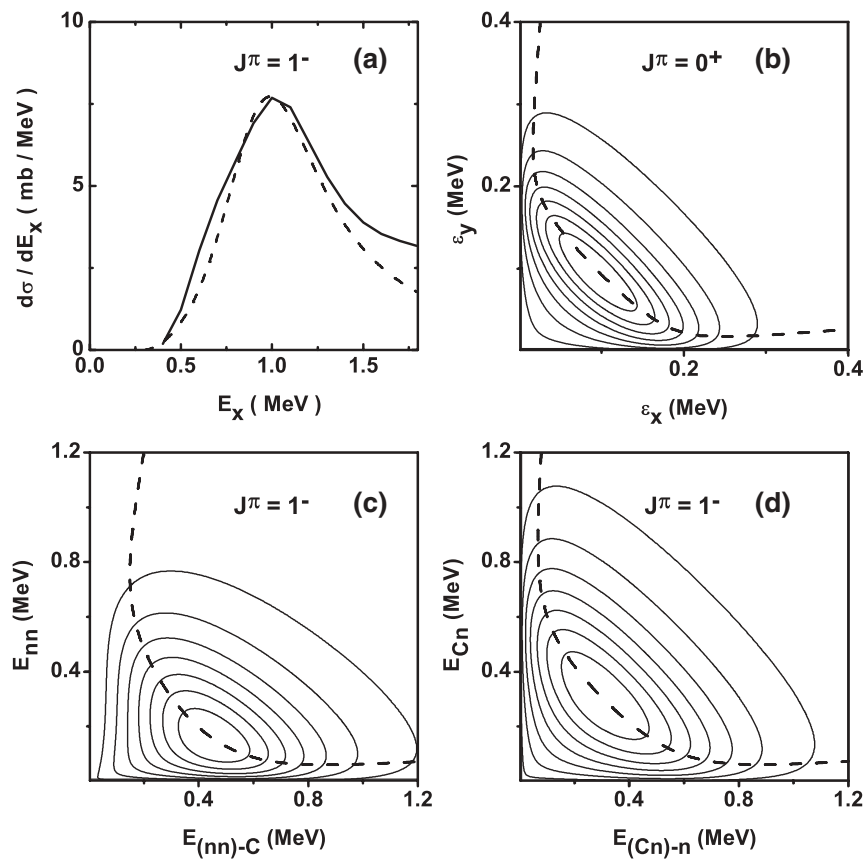

FIG. 1. a) The solid line shows the spectrum of $1^{-}$excitations in ${ }^{11} \mathrm{Li}$ calculated using the P2 model of [17]. The dashed line is the approximation of this spectrum by a three-body resonance shape from Eq. (16) with parameters $E_{0}=1.2 \mathrm{MeV}$ and $\Gamma_{0}=4 \mathrm{MeV}$. The cross section Eq. (15) is calculated for the $0^{+}$(b) and $1^{-}$[(c) in the $\mathbf{T}$ and and (d) in $\mathbf{Y}$ Jacobi systems] bumps in ${ }^{11} \mathrm{Li}$. The dashed line shows the trajectory of the cross section maxima ridge. 
corresponding to the $0^{+}$state in ${ }^{11} \mathrm{Li}$, and the trajectory of the ridge maxima. Figures $1(\mathrm{c})$ and (d) show contour plots and ridges for the $1^{-}$state in the $\mathbf{T}$ and $\mathbf{Y}$ systems, respectively. The corresponding spectrum of the three-body resonance from the approximate formula (16) [dashed line in Fig. 1(a)] is fitted to the calculations (solid line) of the dipole nuclear response obtained in our P2 model [17]. Agreement of ridges in real cross sections with the ridges in Fig. 1 provides an opportunity to judge whether or not a peak in $E$ is a 'true' three-body resonance.

In the following sections using the examples of ${ }^{6} \mathrm{He}$ we shall demonstrate the most important cases of three-body energy correlations:

(i) Energy correlations for narrow three-body resonances;

(ii) Energy correlations for wide three-body resonances;

(iii) Energy correlations for three-body virtual-like excitations.

\section{Binary energy correlations from narrow three-body resonances}

The energy correlations between two particles with corresponding relative motion energy $\epsilon_{x}$, or between a pair and the third particle with relative energy $\epsilon_{y}$, can be obtained after integration of Eq. (15) over the unobserved energy $\epsilon_{y}$ (or $\epsilon_{x}$ ). We wish to determine the general effects on the binary Jacobian energy correlations of a possible low-lying three-body resonance with width $\Gamma_{\mathrm{T}} \ll E_{0}$. Since the resonant denominator is a rapidly varying function we can fix the slowly-varying residual part to its value at a point close to the resonance position $\epsilon_{x}+\epsilon_{y}=E_{0}$, and move it outside the integral. For example, for correlations between particles arising from a narrow three-body resonance, we are left with the dependence

$$
\begin{aligned}
\frac{d \sigma(2)}{d \epsilon_{x}} \propto & \sum_{l_{x} l_{y}}\left(\epsilon_{x}\right)^{l_{x}}\left(E_{0}-\epsilon_{x}\right)^{l_{y}} \sqrt{\epsilon_{x}\left(E_{0}-\epsilon_{x}\right)} \\
& \times \int \frac{d \epsilon_{y i}}{\left(\epsilon_{x i}+\epsilon_{y i}-E_{0}\right)^{2}+\Gamma_{\mathrm{T}}^{2} / 4} .
\end{aligned}
$$

The distribution over $\epsilon_{y}$ is the same after exchanging $x$ and $y$. The integral is equal to $\left(\pi-2 \arctan \left(2\left(\epsilon_{x}-E\right) / \Gamma_{\mathrm{T}}\right)\right) / \Gamma_{\mathrm{T}}$, which varies between $\pi / \Gamma_{\mathrm{T}}$ and $2 \pi / \Gamma_{\mathrm{T}}$.

For ${ }^{6} \mathrm{He},{ }^{11} \mathrm{Li}$ and other Borromean nuclei the natural parity states for the halo neutrons are $\mathrm{J}^{\pi}=0^{+}, 1^{-}, 2^{+}$and lowest possible Jacobian angular momenta should be considered. If $l_{x}=l_{y}$ the distribution is symmetric around the maximum $\epsilon_{x}=E_{0} / 2$, and has characteristic behavior $\epsilon_{x}^{l_{x}+1 / 2}$ at the origin. For $l_{x} \neq l_{y}$, the maximum is located at $\epsilon_{x}=$ $\left(l_{x}+1 / 2\right) E_{0} /\left(l_{x}+l_{y}+1\right)$. At low energy $\epsilon_{x}$ the nonresonant continuum has a shape given by Eq. (17). The binary energy correlations for ${ }^{6} \mathrm{He}$ will be discussed in more detail in the next section.

\section{ENERGY CORRELATIONS IN ${ }^{6} \mathrm{HE}$}

A full range of methods, developed in previous papers to explore three-body continuum structure, has been applied to ${ }^{6} \mathrm{He}$, which is used as a reference case for more complicated halo nuclei. In addition to the sharp $2_{1}^{+}$resonance at $0.8 \mathrm{MeV}$ above the three-body threshold, a second $2_{2}^{+}$resonance was predicted at $2.1 \mathrm{MeV}$ with width $\Gamma \simeq 1.4 \mathrm{MeV}$ (a lower limit since the true width can not be defined because of strong asymmetry), a $1^{+}$resonance at $E=2 \mathrm{MeV}, \Gamma \cong 1.2 \mathrm{MeV}$; and $0^{+}$excitation peaking at $E=1.6 \mathrm{MeV}$ with $\Gamma=1.5 \mathrm{MeV}$. The nature of the so-called "soft dipole mode" suggested in [24], and responsible for an abnormally large electromagnetic dissociation cross-section, still needs more clarification [25]. Various attempts [1,26-34], based on the same cluster representation of ${ }^{6} \mathrm{He}$, have not given a definite answer concerning the existence of a dipole resonance state in ${ }^{6} \mathrm{He}$. The first experimental results, where the dipole response function of ${ }^{6} \mathrm{He}$ in EMD was reconstructed [11], did not show the 1-2 MeV sharp peak in the dipole strength function obtained in some three-body approaches. Recently the experimental binary angular and energy correlations from fragmentation of ${ }^{6} \mathrm{He}$ on $\mathrm{Pb}$ were published [12]. Our theoretical calculations [35] within the microscopic four-body distorted wave model describe well the experimental data for fragments correlations near breakup threshold but with increasing excitation energy of ${ }^{6} \mathrm{He}$ some of the theoretical distributions deviate from experiment.

In early break-up experiments only the momentum distribution of one fragment, or binary correlations (angular, momentum or energy) were observed. As reference cases, the binary channels involving physically significant states, i.e., pronounced resonances or virtual states, are included in the analysis in part $\mathrm{C}$ of this section. Usually they correspond to the knock-out of one of the fragments leaving the other two interacting with small relative energy. In this case the final state wave function is factorized into a relative motion of the two interacting fragments and a nearly plane wave for the third fragment. In the ${ }^{6} \mathrm{He}$ case there are two significant channels: ${ }^{5} \mathrm{He}$ in the $3 / 2^{-}$resonant state and the virtual ${ }^{1} S_{0}$ state in the $n n$ channels. At low excitation energies these channels are coupled and the resulting correlations between fragments will be strongly influenced by channel interplay, leading to a continuum structure differing from a simple sum of binary resonances. Later we will discuss energy correlations: (A) in the g.s., which corresponds to Serber model without final state interaction (NoFSI) for $0^{+}$as well as transitions to other states; and (B) for the transition energy correlations for reactions with full FSI for continuum states.

The part of the nuclear transition operator of Eq. (5) which acts on halo neutrons is used for illustration of correlated responses. The part of Eq. (5), acting on the core [which gives a vanishing cross section by Eq. (2) in the limit of infinitely heavy core] as well as the Coulomb dipole operator give almost similar energy correlations.

In all cases we present three-body and two-body energy correlations, and for all combinations of Jacobi coordinate pairs. In Figs. 2, 3, and 4 below, the lowest natural parity final states for ${ }^{6} \mathrm{He}$ are included. 

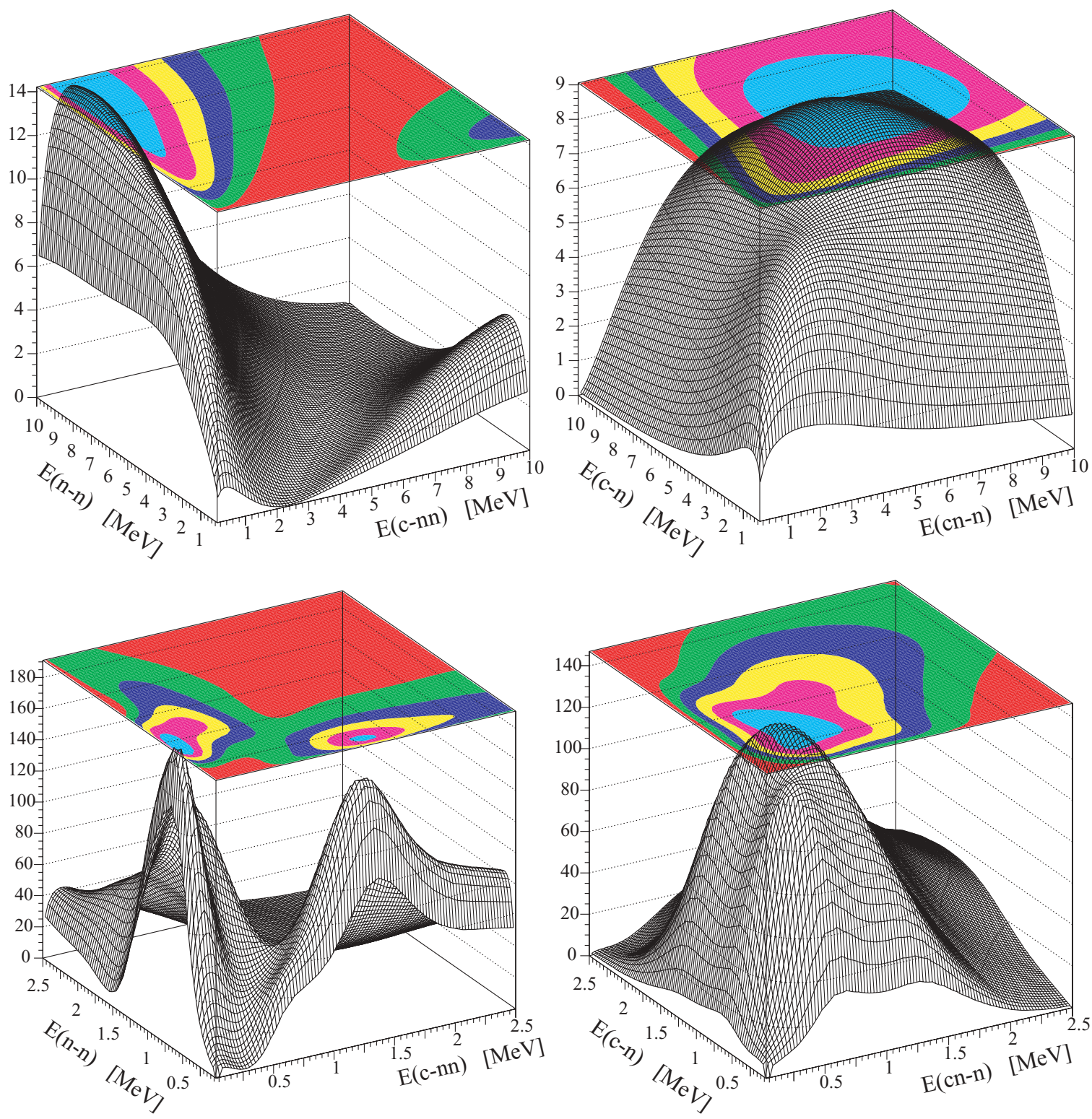

FIG. 2. (Color online) Energy correlation plot (arbitrary units) for $0^{+}$states: transition to antisymmetrized plane wave, i.e., NoFSI (upper row), with FSI (lower row). Left: in cluster T Jacobi system, right: in 'shell-model' Y one. Note the different energy scales of upper and lower plots.

\section{A. NoFSI three-body energy correlations in ${ }^{6} \mathrm{He}$}

NoFSI energy correlations are obtained by putting the antisymmetrized three-body plane wave from Ref. [1] as final state into the expression for the transition amplitude from nuclear responses of Eq. (5).

The correlation plot for the monopole $0^{+}$continuum (upper row in Fig. 2) corresponds to the Serber model (NoFSI in continuum) and demonstrates an important property of the g.s.: the presence of spatial correlations from a 'Pauli focusing' effect $[4,36]$, which generates spatial 'dineutron' and 'cigar' configurations, and correspondingly two peaks in the $E_{n n}-$ $E_{\alpha-(n n)} \mathbf{T}$-system correlation plot.

This clearly reflects the two-dimensional uncertainty principle: the peak at $E_{n n} \sim 10 \mathrm{MeV}$ and small $E_{\alpha-(n n)}$ is a consequence of the 'dineutron' with a small distance between the halo neutrons, while the peak at $E_{n n} \sim 2 \mathrm{MeV}$ and large $E_{\alpha-(n n)}$ is a consequence of the 'cigar'. with a large distance between the neutron pair.

The higher probability of the narrow core- $n n$ energy distribution should to some extent be seen in longitudinal and transverse momentum distributions from fragmentation experiments. The reduced probability of the peak with small $n n$ relative energy, usually thought of as a virtual $n n$ state, arises from the lower 'cigar' peak, where the neutrons have an extreme spatial separation. These correlations in the laboratory frame were studied in detail in Ref. [37].

The NoFSI correlation plot for $\alpha-n$ and $(\alpha n)-n$ energies exhibits a $p_{3 / 2}^{2}$ main bump at $5-6 \mathrm{MeV}$, and an $s_{1 / 2}^{2}$ low energy 

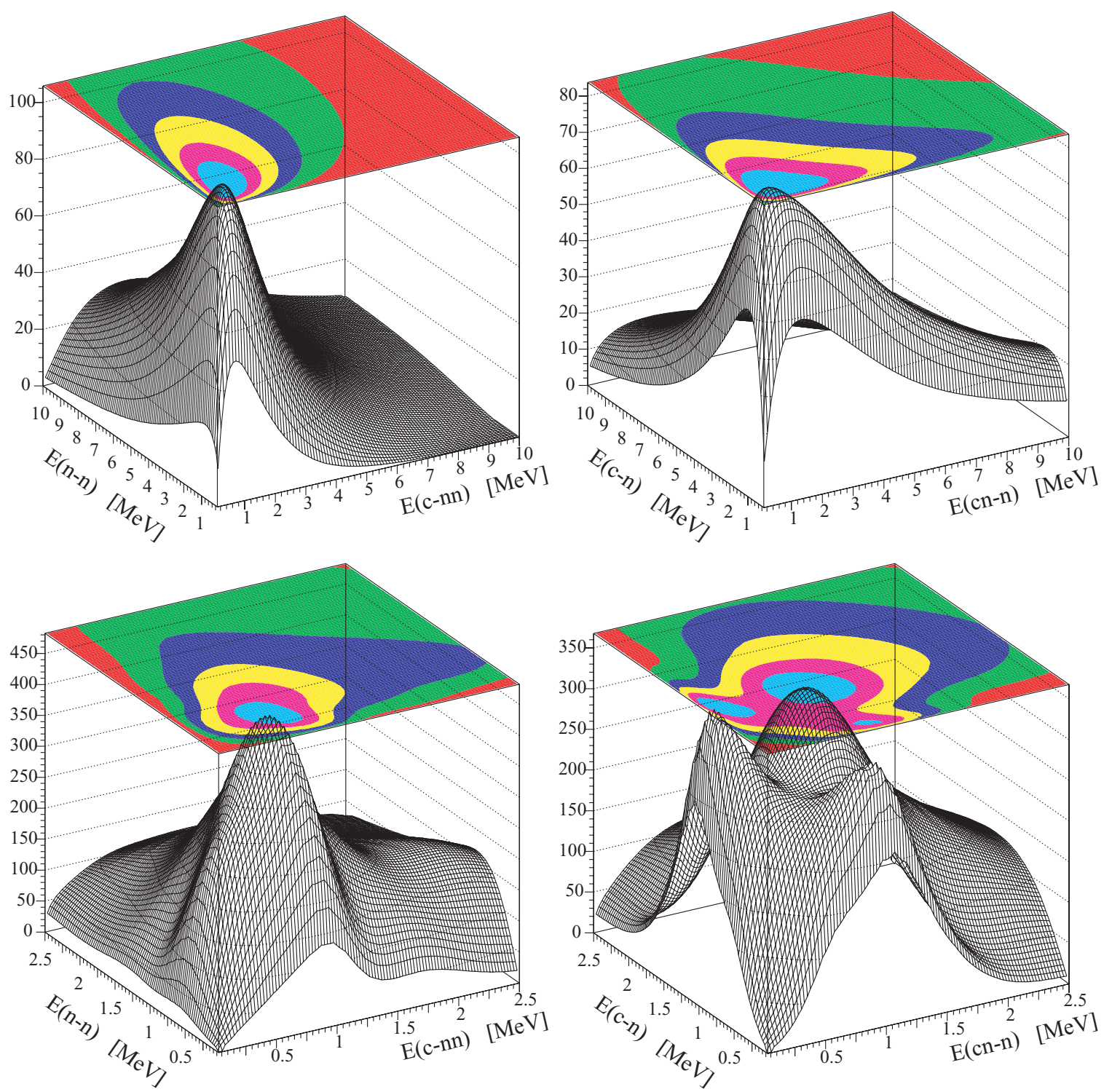

FIG. 3. (Color online) Energy correlation plot (arbitrary units) for $1^{-}$states: transition with antisymmetrized plane wave: no FSI (upper row), with FSI (lower row). Left: in cluster T system, right: in 'shell-model' Y system.

enhancement at about $1-2 \mathrm{MeV}$, according to its small weight in the g.s.

Both $\mathbf{T}$ and $\mathbf{Y}$ correlation plots demonstrate that physically the NoFSI 'mechanism' will give a decay with fast neutrons and a slow $\alpha$-particle. This mechanism gives narrow longitudinal and transverse momentum distributions of the core in fragmentation of ${ }^{6} \mathrm{He}$, but a too wide momentum distribution for the halo neutrons.

For larger multipolarities of the final states, the asymptotics in the classically forbidden region (halo) will play a greater role in the overlap integral Eq. (3) because of centrifugal screening of the internal part of the g.s. wave function in the transition integral.

The correlation plots for the dipole $1^{-}$mode (upper row in Fig. 3) have a remarkable feature: a very pronounced coreneutron ${ }^{5} \mathrm{He}$ correlation at an energy about $1 \mathrm{MeV}$ in both $\alpha-n$ and $(\alpha n)-n$ systems, reflecting however the spatial extent of the g.s. (halo structure). The dipole mode has the best spatial overlap with the g.s. among the $0^{+}, 1^{-}$, and $2^{+}$plane wave continuum states. This can imitate final state interactions, or

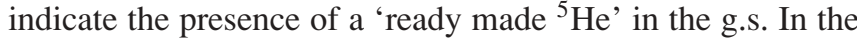
$\mathbf{T}$ system the plot is quite similar to the monopole case with a slow $\alpha$-particle emerging also from cigar-like configuration. Both plots resemble the schematic two-body virtual state case (upper row in Fig. 1) from Ref. [3], but physically the reason is the specific structure of the g.s., and not a resonance in continuum.

The correlation plots for the quadrupole $2^{+}$mode (upper row in Fig. 4) have less pronounced core-neutron ${ }^{5} \mathrm{He}$ correlations, and these are peaked at an energy about $1-2 \mathrm{MeV}$ in both the $\alpha-n$ and $(\alpha n)-n$ partitions. They also reflect the spatial extension of the g.s., but the peak height is smaller than in the dipole case because of the centrifugal screening of the internal part of the g.s. mentioned above. In the $\mathbf{T}$ system there 

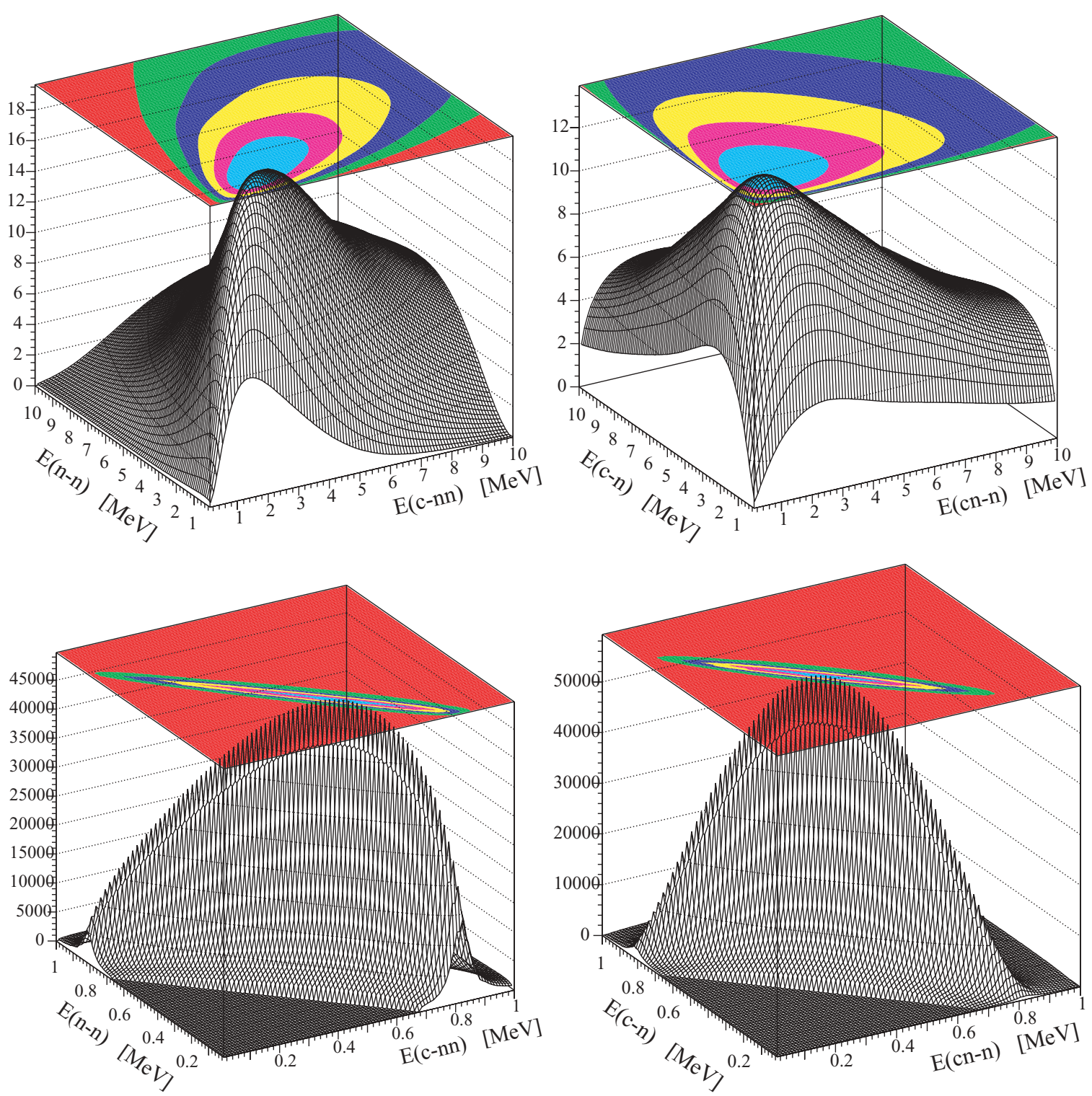

FIG. 4. (Color online) Energy correlation plot (arbitrary units) for $2^{+}$states: transition with antisymmetrized plane wave: NoFSI (upper row), with FSI for the $2_{1}^{+}$resonance (lower row). Left: in cluster $\mathbf{T}$ system, right: in 'shell-model' $\mathbf{Y}$ system.

is a strong amplification of the $s$-wave $E_{n n}$ component which can be seen as a cut along the $E_{c-n n}$ axis in the left upper panel.

\section{B. Transition energy correlations for reactions (FSI)}

\section{Energy correlations for narrow three-body resonances}

The transition correlation plots for the $2_{1}^{+}$resonance in Fig. 4 (lower row) are very similar to the intrinsic correlations in Ref. [3], and are determined by the three-body resonance nature of the final state and by perfectly overlapping radial wave functions for the g.s. and the resonantly amplified internal part of the $2_{1}^{+}$continuum state. The transition to the $2_{1}^{+}$ resonance has an amplitude which is three orders of magnitude greater than that with a three-body plane wave as the final-state and energy correlations with FSI are completely different from the case of NoFSI (upper row).

\section{Energy correlations for wide three-body resonances}

The second $2_{2}^{+}$resonance is shown in Fig. 5. It exhibits a three-body resonant-like structure in contrast to the intrinsic energy correlations in Ref. [3] where only the $s$-wave $n-n$ virtual state stands out. The reason is that the halo g.s. plays the role of a filter, cutting off impact parameters which correspond to hypermoments $K>2$ and selects the lowest $K=2$ in which the resonance still exists. In the $\mathbf{T}$ system it has an asymmetric shape due to the strong influence of the virtual $n n$ correlation. Since the $2_{2}^{+}$resonance state has a different Young tableau symmetry from the g.s., the transition probability integrated over the resonance region is about 10 times less 


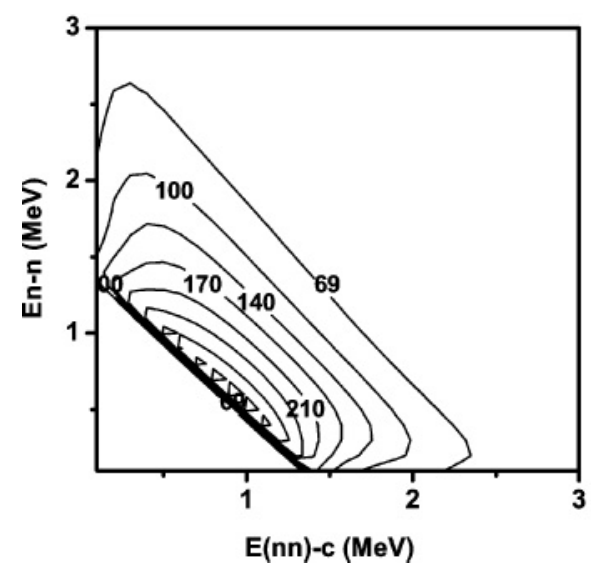

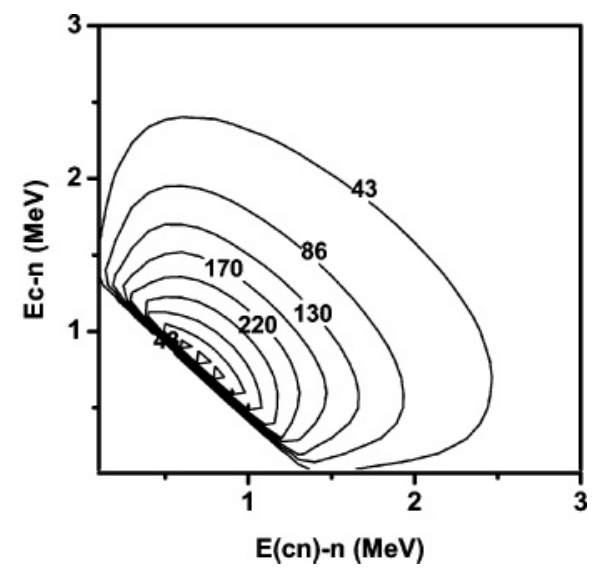

FIG. 5. (Color online) Transition energy correlation plot (arbitrary units) for a $2_{2}^{+}$resonance state: Left: in cluster $\mathbf{T}$ system, right: in 'shell-model' Y system. than for $2_{1}^{+}$, which has approximately the same Young tableau symmetry as the g.s. The maxima ridge line $\epsilon_{x}+\epsilon_{y}=1.5$ $\mathrm{MeV}$ corresponds in both $\mathbf{T}$ and $\mathbf{Y}$ systems to the peak in the response function and also to a resonant behavior in the eigenphase, crossing $\pi / 2$ at energy of about $2 \mathrm{MeV}$ for $2_{2}^{+}$. This visible difference in the maxima ridge compared with Fig. 10 from our previous paper [3] is due to interference with background in $3 \rightarrow 3$ scattering in [3].

The correlated response for the $1^{+}$resonance, shown by a contour plot in Fig. 6, exhibits a three-body resonant structure quite similar to $2_{2}^{+}$. The cutoff by the g.s. size filter selects also here the resonant hypermoment $K=2$. For the same reason, the transition probability integrated over the resonance region is again an order of magnitude less than for $2_{1}^{+}$. The ridge line $\epsilon_{x}+\epsilon_{y}=2.8 \mathrm{MeV}$ in both $\mathbf{T}$ and $\mathbf{Y}$ systems has a corresponding line in the intrinsic correlation plot [3]. The peak in the response function and the resonant behavior are again due to two almost coinciding eigenphases, of different physical origin, which cross $\pi / 2$ at energies around 2.4 MeV.

\section{Energy correlations for three-body virtual-like excitations}

The monopole $0^{+}$transition correlations (Fig. 2) reveal a strong combined influence of FSI in the continuum and of the g.s. structure. Since for a zero-range nuclear transition operator the response is zero due to orthogonality of the g.s. and continuum wave functions, a finite range transition operator has been used. Hyperradial matrix elements of any short-range operator (i.e., decreasing faster than exponentially) have the universal behavior $1 / \rho^{3}$ in the monopole case. We use a form factor $1 /\left(1+\left(\rho / \rho_{0}\right)^{3}\right)$ with range $\rho_{0}=5 \mathrm{fm}$. The partial composition of the wave functions is about $80 \%$ spin $S=0$ with dominant zero orbital angular momenta in T, and about $18 \%$ spin $S=1$ with orbital angular momenta $l_{x}=l_{y}=1$. Comparison with the g.s. energy correlations (NoFSI case in Fig. 2) shows similarities in the general behavior. Both have two wings in the $\mathbf{T}$ system, reflecting the g.s., but the scales of the wings in the $\mathbf{T}$ system and of the peak in $\mathbf{Y}$ are larger by orders of magnitude for FSI. Figure 2 shows that in the $\mathbf{T}$ system the g.s. energy correlations have peak positions at $E_{n-n}=9 \mathrm{MeV}, E_{\alpha-n n}=1.5$ $\mathrm{MeV}$ and $E_{n-n}=1.5 \mathrm{MeV}, E_{\alpha-n n}=13 \mathrm{MeV}$ while for FSI transition correlations $E_{n-n}=0.8 \mathrm{MeV}, E_{\alpha-n n}=0.15 \mathrm{MeV}$ and $E_{n-n}=0.15 \mathrm{MeV}, E_{\alpha-n n}=1.3 \mathrm{MeV}$. The FSI peak at $E_{n-n}=0.8 \mathrm{MeV}, E_{\alpha-n n}=0.15 \mathrm{MeV}$ (its narrow part) comes from intrinsic continuum properties (Fig. 6 in Ref. [3]), and also generally reflects an interplay of the g.s. ('dineutron' and 'cigar' spatial configurations) and the FSI in the continuum, enhancing the soft part of spectrum. In the $\mathbf{Y}$ system there are peaks at $E_{n-\alpha}=7 \mathrm{MeV}, E_{n \alpha-n}=7 \mathrm{MeV}$ (g.s. induced)

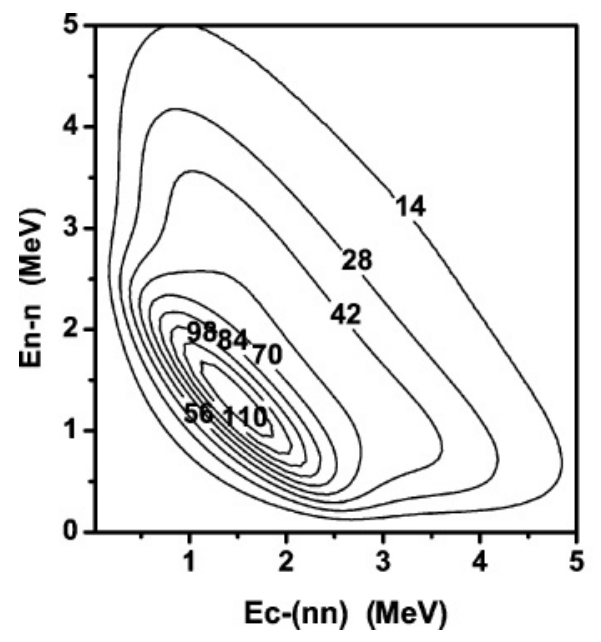

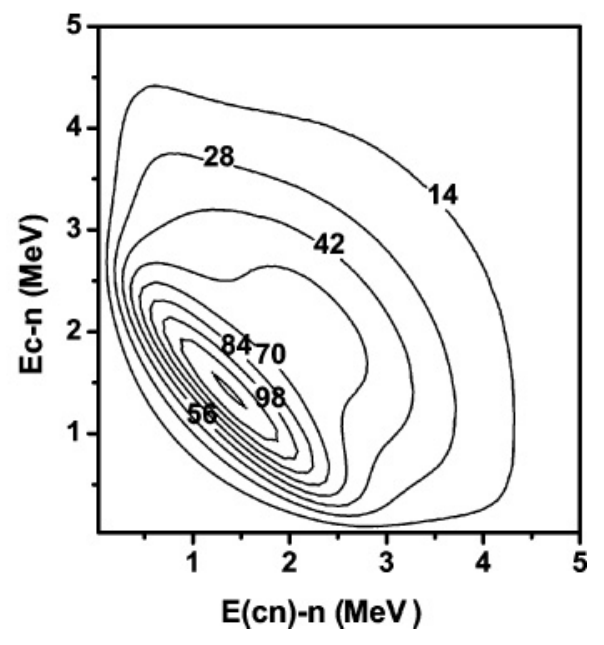

064612-10
FIG. 6. (Color online) Transition energy correlation plot (arbitrary units) for a $1^{+}$resonance state: Left: in cluster $\mathbf{T}$ system, right: in 'shell-model' $\mathbf{Y}$ system. 
and $E_{n-\alpha}=0.8 \mathrm{MeV}, E_{n \alpha-n}=0.6 \mathrm{MeV}$ (due to FSI in continuum). A low energy enhancement at $E_{n-\alpha}=0.4 \mathrm{MeV}$, $E_{n \alpha-n}=0.4$ coincides with an analogous peak for intrinsic continuum correlations and reflects their influence.

The largest g.s. and continuum components $K=2, l_{x}=$ $0, l_{y}=0$ in the $\mathbf{T}$ system give $80 \%$ of the peak value, and define the same 'dineutron' (small $E_{n-n}$ ) and 'cigar' (small $E_{\alpha-n n}$ ) correlations. As was expected, large correlation distances $\sim 15-20$ fm from $K=6-10$ in the continuum are suppressed by the limited size of the g.s., and the second bump in the $\mathbf{T}$ system at $\sim 3 \mathrm{MeV}$ (which appears for intrinsic continuum correlations [3]), is not seen in FSI transitions. The largest component $K=2, l_{x}=0, l_{y}=0$ in the $\mathbf{T}$ basis corresponds almost completely to $K=2, l_{x}=1, l_{y}=1$ in the $\mathbf{Y}$ system, giving a one-peak structure.

In the $\mathbf{T}$ system, correlations at small $E_{\alpha-n n}$ and $E_{n-n}$ are caused by $s$-waves in accordance with the dominating component $K=2, l_{x}=0, l_{y}=0$, which gives a correlated response

$$
\frac{d B}{d E_{\alpha-n n} d E_{n-n}} \propto\left(\frac{E_{\alpha-n n}-E_{n-n}}{E}\right)^{2} \sqrt{E_{\alpha-n n} E_{n-n}} .
$$

In the $\mathbf{Y}$ system, correlations at small $E_{n-\alpha}$ and $E_{n \alpha-n}$ have a largely $p$-wave behavior with rather small $s$-wave contribution. This gives a correlated response

$$
\frac{d B}{d E_{n-\alpha} d E_{n \alpha-n}} \propto \frac{E_{n-\alpha} E_{n \alpha-n}}{E^{2}} \sqrt{E_{n-\alpha} E_{n \alpha-n}} .
$$

The dipole $1^{-}$transition correlations shown in Fig. 3 have structure that reflects the dominance of singlet $n$ - $n s$-wave motion (about $85 \%$ ) in $\mathbf{T}$. Two nearly symmetric peaks at $1 \mathrm{MeV}$ in $\mathbf{Y}$ (in $n-\alpha$ and ( $n \alpha)-n$ subsystems) are due to the $n n$ antisymmetrization in the main $s \otimes p$ and $d \otimes p$ orbital components, and their interference. At small energies $s$-motion prevails in both subsystems. The double-bell structure comes from $K=3, l_{x}=0, l_{y}=1$ and is symmetric in $l$ components, while the valley between them is due to $K=1, l_{x}=0, l_{y}=1$ mostly. The peak at $E_{\alpha-n n} \sim E_{n-n} \sim 0.8 \mathrm{MeV}$ is the memory of the g.s. spatial structure, which is seen in the g.s. NoFSI correlations plot in the same figure.

Physically there are two main decay channels having the highest matching with the g.s. The first is the $p_{3 / 2}$ 'ground state' of ${ }^{5} \mathrm{He}$, for which the internal part of the wave function is amplified in a compact region with large overlap with the g.s. of ${ }^{6} \mathrm{He}$. It is also kinematically favorable that the second neutron is in an $s_{1 / 2}$ state with zero centrifugal barrier. The second channel is the singlet $n-n s$-wave virtual state, in cluster $\mathbf{T}$ coordinates with relative orbital angular momentum $l_{\alpha-(n n)}=1$ due to dipole transition from the ${ }^{6} \mathrm{He}$ g.s., which has $85 \% l_{n n}=0, l_{\alpha-(n n)}=1$ component. Therefore components with $l_{x}=0, l_{y}=1$ and hypermoments $K \leqslant 3$ will dominate the dipole transition.

In the $\mathbf{T}$ basis only one peak in $n-n$ relative motion appears at $0.8 \mathrm{MeV}$. This peak also comes from $K=1-3$ with a characteristic $s$-wave shape at small energies. But the higher energy intrinsic correlations, coming from $K=5-11$, and correspond to a separation between the neutrons of about 16-20 fm, have disappeared in transition correlation due to the significantly smaller size of the g.s., which cuts the long distance peaked continuum components, pushed out by high centrifugal barriers.

In the $\mathbf{T}$ system, correlations at small $E_{\alpha-n n}$ and $E_{n-n}$ are caused by dominant $s$-wave $n n$ motion $\left[K=1(3), l_{x}=\right.$ $\left.0, l_{y}=1\right]$, which gives a correlated response proportional to

$$
\frac{d B}{d E_{\alpha-n n} d E_{n-n}} \propto \frac{E_{\alpha-n n}}{E} \sqrt{E_{\alpha-n n} E_{n-n}} .
$$

In the $\mathbf{Y}$ the Raynal-Revai transform gives a superposition $\left(l_{x}=1, l_{y}=0\right)-b\left(l_{x}=0, l_{y}=1\right)$. Therefore the correlations at small $E_{n-\alpha}$ and $E_{n \alpha-n}$ have a mostly $s$-wave character because of antisymmetrization constraints. This gives a correlated response

$$
\frac{d B}{d E_{n-\alpha} d E_{n \alpha-n}} \propto \frac{\left(\sqrt{E_{n-\alpha}}-b \sqrt{E_{n \alpha-n}}\right)^{2}}{E} \sqrt{E_{n-\alpha} E_{n \alpha-n}}
$$

with $b \neq 1$ due to the recoil effect, and $b \rightarrow 1$ for increasing core mass.

\section{Two-body energy correlations in ${ }^{6} \mathrm{He}$}

The two-body correlations depicted in Fig. 7 are obtained by integration of the three-body correlated responses over an unobserved pair energy. For all final states with natural parity, the distributions for both $n-n$ and $\alpha-n$ are different from what they would be without final state binary interactions, which can be seen from the significant change of peak positions for NoFSI versus FSI three-body correlated responses.

An effective mechanism that can impede binary FSI can be illustrated by the example of the $2_{1}^{+}$resonance at $E_{0}=0.8 \mathrm{MeV}$ in ${ }^{6} \mathrm{He}$. If we examine the leading $p_{3 / 2} p_{3 / 2}$ component in the $\mathbf{Y}$ system, where the $p_{3 / 2}$ resonance in ${ }^{5} \mathrm{He}$ is located at about the same energy, $E_{n-\alpha} \sim 0.8 \mathrm{MeV}$, we can see from the correlation plot Fig. 4 for a narrow resonance, that due to limitation by the available phase space $E_{n-\alpha}+E_{n \alpha-n}=E_{0}$ set by the narrow resonance position $E_{0}$ and an almost equal sharing of the energy by the $\alpha-n$ and $(\alpha n)-n$ subsystems, the formation of ${ }^{5} \mathrm{He}$ will be suppressed.

In the absence of a narrow three-body resonance, as in transitions to the $0^{+}$and $1^{-}$continuum states, the shape of $\alpha-n$ distributions is defined by the interplay of different configurations in the region of interaction, and by the possible channels of decay. For the most interesting case, that of the $1^{-}$dipole mode, the correlation plots for the full FSI, NoFSI and a free binary $(\alpha-n) p_{3 / 2}$ interaction $\left({ }^{5} \mathrm{He}\right.$ '), i.e., with a noninteracting third neutron, are shown in Fig. 8. The sharply peaked ${ }^{5} \mathrm{He}$ correlation curve is scaled down by a factor of three to demonstrate the deviation from the full FSI case. Due to antisymmetrization it is impossible to divide the distribution into $p_{3 / 2}$ and $s_{1 / 2}$ parts since both halo neutrons spend "half time" in each. The soft (lower) part of the full FSI distribution has $s$-wave nature, and both the virtual $s$-state in the $n-n$ subsystem and the $s_{1 / 2}$ partial state in $\alpha-n$ subsystem are 

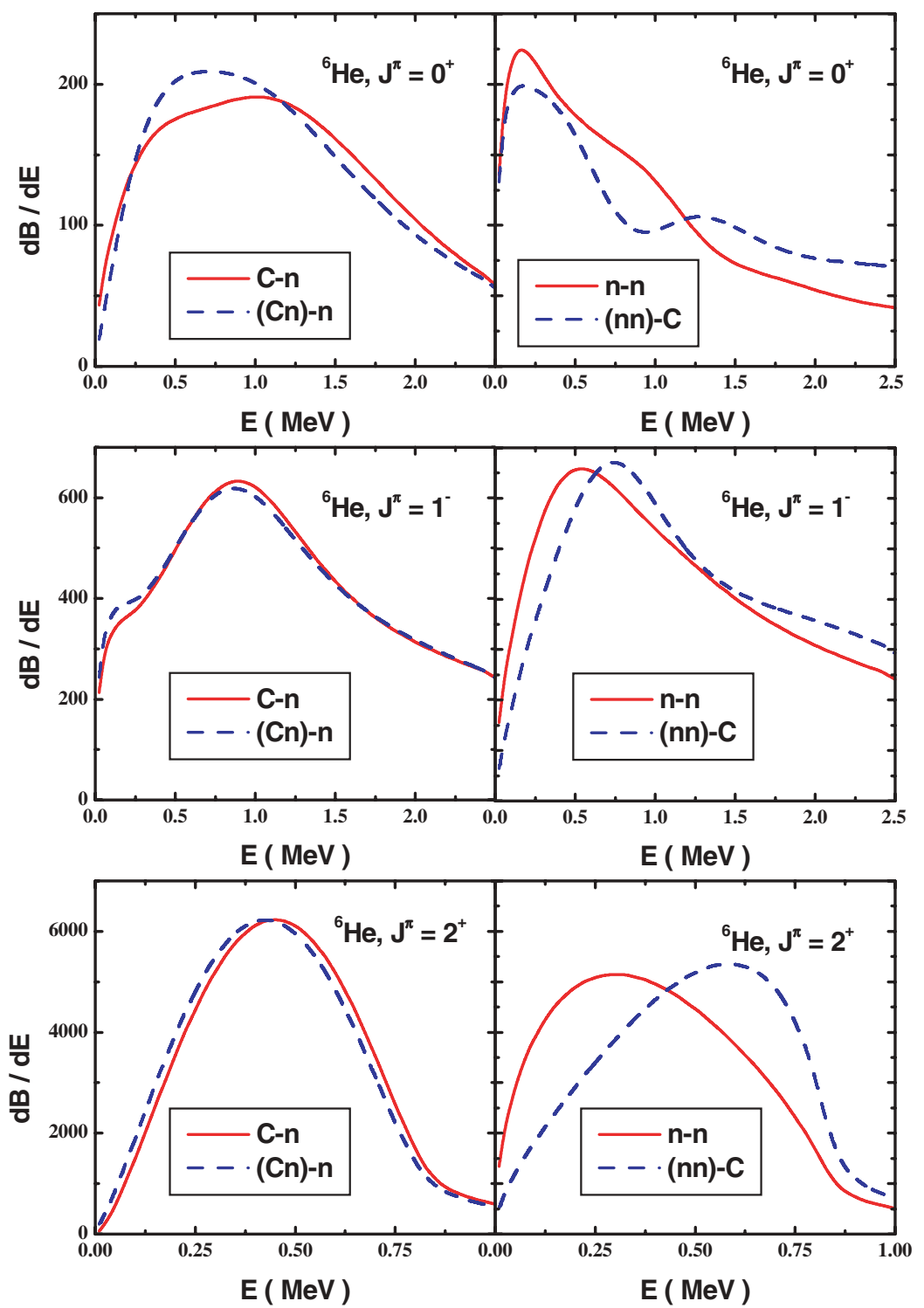

FIG. 7. (Color online) Two-body nuclear type transition correlations (arbitrary units) for natural parity continuum final states in ${ }^{6} \mathrm{He} ; n-n$ is between two halo neutrons, C- $n$ is between core $(\alpha)$ and neutron, $(\mathrm{Cn})-n$ is between the centre of mass of the $\mathrm{C}+n$ subsystem and the neutron, $(n n)-\mathrm{C}$ is between the center of mass of the $n+n$ subsystem and the core. (Note that $\mathbf{T}$ is now in the right column while $\mathbf{Y}$ is in the left.)

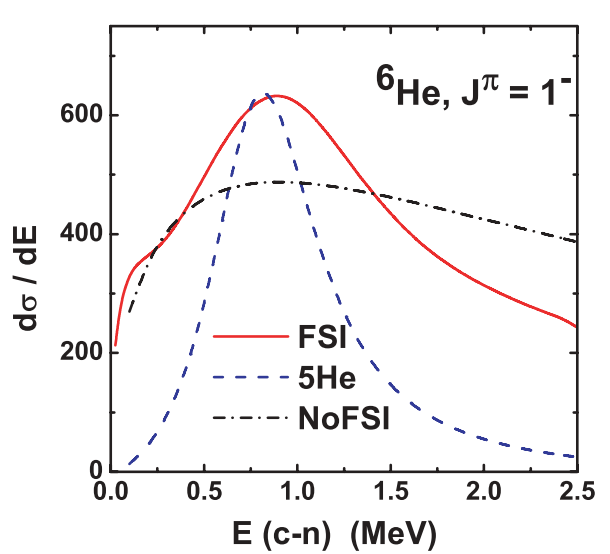

FIG. 8. (Color online) Two-body nuclear type transition correlations (arbitrary units) between core $(\alpha)$ and a halo neutron for $1^{-}$ final continuum states in ${ }^{6} \mathrm{He}$. The solid line represents full FSI in the $1^{-}$continuum, the dashed line represents the $p_{3 / 2}$ interaction between core $(\alpha)$ and neutron constituent ( ${ }^{5} \mathrm{He}$ ') without interaction with the last halo neutron, and the dash-dotted line is NoFSI in the $1^{-}$continuum. responsible for that. At higher energy there is combined action of higher partial waves. The position of the (flat) maximum in the NoFSI case is defined exclusively by the geometry of the halo g.s. Its position coincides best with the FSI peak position providing evidence that the halo g.s. and not the decay via ${ }^{5} \mathrm{He}$ is responsible also for the FSI $\alpha-n$ energy correlation, which is influenced only mildly by attraction in the $1^{-}(\alpha-n-n)$ system as a whole [1]. This enhances the soft part of the spectrum.

The three $\mathbf{T}$-system correlation plots on the right hand side of Fig. 7, for different multipolarities in the final state, demonstrate the effect of the third body on the $n-n$ interaction. Only for transition to the $0^{+}$continuum state, where the position of the $n-n$ peak is lowest in energy, does the shape of the correlation function differ slightly from that of free $n n$ interaction. This is in agreement with the Migdal-Watson formula [38] for two-neutron decay.

In Ref. [10] the results of calculations for two-body energy correlations in ${ }^{6} \mathrm{He}$ breakup within our four-body DWIA theory were presented, and comparison made with GSI experiment [11]. 


\section{CONCLUSION}

Different aspects of the three-body continuum were outlined and discussed in our previous papers [1-3]. In this paper we have concentrated on the three-body energy correlations in the nuclear type transitions from the g.s. to the three-body continuum using again ${ }^{6} \mathrm{He}$ (and sometimes ${ }^{11} \mathrm{Li}$ ) as the test case for Borromean systems. These transitions are the main building blocks in one-step reaction theory where the transition operators intertwine the properties of the bound state with the intrinsic correlated structure of the three-body continuum. The bumps in the excitation spectrum, generated by three-body break-up reactions of diffraction type, have four possible sources: (i) true three-body resonances; (ii) Efimov-like (or virtual-like) continuum structures; (iii) long lived binary resonance in one of the pairs; (iv) response of an extended system to long-range transition operators. To reach definite conclusions about the nature of resonant amplification it is necessary to inspect two types of correlations: the correlations in the g.s. only (or no final state interactions), and transition energy correlations with all final state interactions included. The NoFSI is used in sudden approximation (or to some extent in the Serber break-up model), while the FSI is probed by nuclear and electromagnetic responses.

The uncertainty principle and halo structure of the bound state are mostly responsible for the momentum (and energy) correlations in the g.s. and could be reflected in the specific conditions of transverse and longitudinal momentum distributions in high-energy fragmentation reactions.

The spatial structure of the FSI continuum wave functions [2] shows a behavior in the internal region that deviates markedly from that of a three-body plane wave with antisymmetrized halo neutrons. Thus a narrow three-body resonant state is characterized by a localization in the region of mutual interaction of all three particles, and therefore by a strong amplification of the interior part of wave function in this region. Due to high radial spatial overlap with the g.s. (or with the reaction volume in the general case) the transitions to these states are very strong. The $S$-matrix poles coincide with the peak energy in a resonant amplification of the interior part of the wave function, and the intrinsic energy-momentum correlations for 3-3 scattering almost coincide with the transition energy correlations for reactions. The main criterion for the existence and properties of any intrinsic resonant state is that they should not depend on the excitation mechanism. This serves as a signature for the three-body resonant nature of an observed resonant-like enhancement in cross sections. We have shown that these enhancements are revealed in the structure of the energy correlation functions, and are most pronounced for the $2_{1}^{+}$ resonance in ${ }^{6} \mathrm{He}$, less so for the wider $2_{2}^{+}$and $1_{1}^{+}$resonances.

The origins of Efimov-like continuum structures are longrange effective interactions between the three bodies with range of the order of the sum of the scattering lengths in the binary subsystems. These are responsible for the bound state Efimov effect [18], and for the compression of continuum spectra near the three-body threshold. Their pronounced inherent characteristic - of a very long range of formation-implies a large number of configurations in an $\mathrm{HH}$ basis, a lack of pockets in the diagonal potential terms, and strong off-diagonal (coupling) terms. A signal for possible existence of Efimov-like 'three-body virtual' excitations is the presence of virtual states and low-lying resonances in the binary subsystems.

When we deal with a Borromean halo, the neutron-neutron interaction with scattering length $\sim 16 \mathrm{fm}$ is decisive for this effect ('continuum pairing'). In this case there will be no concentration of the wave function inside the region of interaction of all particles, but a long range spreading of correlations, and the transition matrix element will have overlap of the bound state with a small fraction of the correlated part of the continuum wave function. Therefore the intrinsic correlated structure of the three-body continuum itself, discussed in Ref. [3], will sometimes be strongly filtered by the g.s. structure.

Our exploration of the binary energy correlations ( $n-n$ and $\alpha-n)$ in ${ }^{6} \mathrm{He}$ has shown a complex interplay of the $s$-wave $n-n$ virtual state and the $p_{3 / 2}$ resonance in the $\alpha-n$ subsystem $\left({ }^{5} \mathrm{He}\right)$, both for monopole and dipole soft modes. In most cases these correlations differ strongly from "free" binary correlations since the presence of the third body in FSI is decisive.

We have shown that in three-body correlations for all configurations, in spite of presence of a soft dipole resonance-like peak in both nuclear and electromagnetic response functions at $\sim 1.5 \mathrm{MeV}$ [1], there is lack of noticeable true resonant behavior in the interior region, and the same applies for the monopole case.

Thus we have demonstrated that transition energy correlations serve as a useful tool for a detailed theoretical analysis in three-body continuum spectroscopy. This tool will in forthcoming papers also be used for the case of ${ }^{11} \mathrm{Li}$ where the ground state is a strong mixture of $p^{2}$ and $s^{2}$ motions, adding new features to the Borromean continuum structure as well.

\section{ACKNOWLEDGMENTS}

This work was done with financial support from Bergen and UK from the EPSRC grants GR/R/25514 and GR/M/82141. B.V.D. and S.N.E. acknowledge support from RFBR grants 05-02-17535 and 1885.2003.2. Part of the work was performed under the auspices of the University of California, Lawrence Livermore National Laboratory under contract No. W-7405Eng-48. The authors are grateful to Prof. B. Jonson and Dr. G. Hagen for useful discussions.
[1] B. V. Danilin and M. V. Zhukov, Sov. J. Nucl. Phys. 56, 460 (1993); B. V. Danilin, I. J. Thompson, J. S. Vaagen, and M. V. Zhukov, Nucl. Phys. A632, 383 (1998).
[2] B. V. Danilin, T. Rogde, J. S. Vaagen, I. J. Thompson, and M. V. Zhukov, Phys. Rev. C 69, 024609 (2004). 
[3] B. V. Danilin, J. S. Vaagen, T. Rogde, S. N. Ershov, I. J. Thompson, and M. V. Zhukov, Phys. Rev. C 73, 054002 (2006).

[4] M. V. Zhukov, B. V. Danilin, D. V. Fedorov, J. M. Bang, I. J. Thompson, and J. S. Vaagen, Phys. Rep. 231, 151 (1993).

[5] B. V. Danilin, M. V. Zhukov, A. A. Korsheninnikov, L. V. Chulkov, V. D. Efros, Sov. J. Nucl. Phys. 49, 351, 359 (1989); 53, 71 (1991); B. V. Danilin, M. V. Zhukov, S. N. Ershov, F. A. Gareev, R. S. Kurmanov, J. S. Vaagen, and J. M. Bang, Phys. Rev. C 43, 2835 (1991).

[6] D. Gogny, P. Pires, and R. de Tourreil, Phys. Lett. B32, 591 (1970).

[7] S. N. Ershov, T. Rogde, B. V. Danilin, J. S. Vaagen, I. J. Thompson, and F. A. Gareev, Phys. Rev. C 56, 1483 (1997).

[8] S. N. Ershov, B. V. Danilin, T. Rogde, and J. S. Vaagen, Phys. Rev. Lett. 82, 908 (1999).

[9] S. N. Ershov, B. V. Danilin, J. S. Vaagen, A. A. Korsheninnikov, and I. J. Thompson, Phys. Rev. C 70, 054608 (2004).

[10] S. N. Ershov, B. V. Danilin, and J. S. Vaagen, Phys. Rev. C 64, 064609 (2001).

[11] T. Aumann et al., Phys. Rev. C 59, 1252 (1999).

[12] L. V. Chulkov et al., Nucl. Phys. A759, 23 (2005).

[13] T. Kraemer et al., Nature (London) 440, 315 (2006).

[14] M. V. Zhukov and B. Jonson, Nucl. Phys. A589, 1 (1995).

[15] M. V. Zhukov, L. V. Chulkov, D. V. Fedorov, B. V. Danilin, J. M. Bang, J. S. Vaagen, and I. J. Thompson, J. Phys. G 20, 201 (1994).

[16] H. Feshbach, Theoretical Nuclear Physics; Nuclear Reactions (Wiley, New York, 1992), p. 162.

[17] I. J. Thompson and M. V. Zhukov, Phys. Rev. C 49, 1904 (1994).

[18] V. Efimov, Comments Nucl. Part. Phys. 19, 271 (1990).

[19] I. J. Thompson, B. V. Danilin, V. D. Efros, M. V. Zhukov, and J. S. Vaagen, J. Phys. G 24, 1505 (1998).

[20] A. M. Badalyan, L. P. Kok, M. I. Polikarpov, and Yu. A. Simonov, Phys. Rep. 82, 31 (1982).

[21] A. I. Baz', Zh. Eksp. Teor. Fiz. 70, 397 (1976).
[22] J. Raynal and J. Revai, Nuovo Cimento A 68, 612 (1970).

[23] A. I. Baz' and S. P. Merkuriev, Theor. Mat. Phys. 70, 397 (1976).

[24] P. G. Hansen and B. Jonson, Europhys. Lett. 4, 409 (1987); K. Ikeda, INS report JHP-7 (1988) [in Japanese].

[25] A. C. Hayes and S. M. Sterbenz, Phys. Rev. C 52, 2807 (1995); A. Csótó, ibid. 52, 2809 (1995).

[26] B. V. Danilin, M. V. Zhukov, J. S. Vaagen, and J. M. Bang, Phys. Lett. B302, 129 (1993).

[27] A. Csótó, Phys. Lett. B315, 24 (1993); Phys. Rev. C 48, 165 (1993); 49, 3035, 2244 (1994).

[28] S. Funada, H. Kameyama, and Y. Sakuragi, Nucl. Phys. A575, 93 (1994).

[29] K. Arai, Y. Suzuki, and K. Varga, Phys. Rev. C 51, 2488 (1995); K. Arai, Y. Suzuki, and R. G. Lovas, ibid. 59, 1432 (1999).

[30] S. Aoyama, S. Mukai, K. Kato, and K. Ikeda, Prog. Theor. Phys. 93, 99 (1995); 94, 343 (1995).

[31] G. F. Filippov, K. Kato, and S. V. Korennov, Prog. Theor. Phys. 96, 575 (1996).

[32] J. M. Bang, A. I. Mazur, A. M. Shirokov, Yu. F. Smirnov, and S. A. Zaytsev, Ann. Phys. (NY) 280, 299 (2000).

[33] A. Cobis, D. V. Fedorov, and A. S. Jensen, Nucl. Phys. A631, 793c (1998); Phys. Rev. C 58, 1403 (1998); E. Garrido, D. V. Fedorov, and A. S. Jensen, Nucl. Phys. A650, 247 (1999).

[34] B. S. Pudliner, V. R. Pandharipande, J. Carlson, S. C. Pieper, and R. B. Wiringa, Phys. Rev. C 56, 1720 (1997); R. B. Wiringa, Nucl. Phys. A631, 70c (1998).

[35] S. N. Ershov, B. V. Danilin, and J. S. Vaagen, Phys. Rev. C 74, 014603 (2006).

[36] B. V. Danilin, M. V. Zhukov, A. A. Korsheninnikov, L. V. Chulkov, and V. D. Efros, Sov. J. Nucl. Phys. 48, 1208 (1988).

[37] M. V. Zhukov, L. V. Chulkov, B. V. Danilin, and A. A. Korsheninnikov, Nucl. Phys. A533, 428 (1991).

[38] K. M. Watson, Phys. Rev. 88, 1163 (1952); A. B. Migdal, Sov. Phys. JETP 1, 2 (1955). 